\title{
Chromatic perception of non-invasive lighting of cave paintings
}

Jesús Zoido, Daniel Vazquez, Antonio Álvarez, Eusebio Bernabeu, Ángel García, et al.

Jesús Zoido, Daniel Vazquez, Antonio Álvarez, Eusebio Bernabeu, Ángel García, Juán Antonio Herraez, Marian del Egido, "Chromatic perception of non-invasive lighting of cave paintings," Proc. SPIE 7429, Novel Optical Systems Design and Optimization XII, 742907 (22 August 2009); doi: 10.1117/12.826503

SPIE Event: SPIE Optical Engineering + Applications, 2009, San Diego, California, United States 


\title{
Chromatic perception of non-invasive lighting of cave paintings
}

\author{
Jesús Zoido*a , Daniel Vazquez ${ }^{\mathrm{a}}$, Antonio Álvarez ${ }^{\mathrm{a}}$, Eusebio Bernabeu ${ }^{\mathrm{b}}$, Ángel García ${ }^{\mathrm{c}}$, Juán Antonio \\ Herraez $^{\mathrm{d}}$, Marian del Egido ${ }^{\mathrm{d}}$ \\ ${ }^{a}$ Departmento de Óptica, Universidad Complutense de Madrid, Escuela Universitaria de Óptica, \\ C/Arcos de Jalón s/n, 28037 Madrid, Spain; \\ ${ }^{\mathrm{b}}$ Departmento de Óptica, Universidad Complutense de Madrid, Facultad de Ciencias Físicas, Ciudad \\ Universitaria s/n, 28040 Madrid, Spain; \\ ${ }^{c}$ Departamento de Física Aplicada a los Recursos Naturales, Universidad Politécnica de \\ Madrid, Escuela Técnica Superior de Ingenieros de Montes, Ciudad Universitaria s/n, 28040 Madrid, \\ Spain; \\ ${ }^{\mathrm{d}}$ Instituto del Patrimonio Cultural de España, C/El Greco n ${ }^{\circ} 4$ (Ciudad Universitaria) 28040 \\ Madrid, Spain
}

\begin{abstract}
This work is intended to deal with the problems which arise when illuminanting Paleolithic cave paintings. We have carried out the spectral and colorimetric characterization of some paintings located in the Murcielagos (bats) cave (Zuheros, Córdoba, Spain). From this characterization, the chromatic changes produced under different lighting conditions are analysed. The damage function is also computed for the different illuminants used. From the results obtained, it is proposed an illuminant whose spectral distribution diminishes the damage by minimizing the absorption of radiation and optimises the color perception of the paintings in this cave. The procedure followed in this study can be applied to optimise the lighting systems used when illuminating any other art work
\end{abstract}

Keywords: Lighting rock art, Color in the art, applied Colorimetry, Lighting cultural heritage

\section{INTRODUCTION}

The conservation of the cultural heritage and its associated artistic production raises two major problems. On the one hand, it is necessary to exhibit the artistic production which conforms the historical patrimony of a country and show it to the public. This fact imposes the necessity to use proper sources of lighting in order to achieve an adequate perception

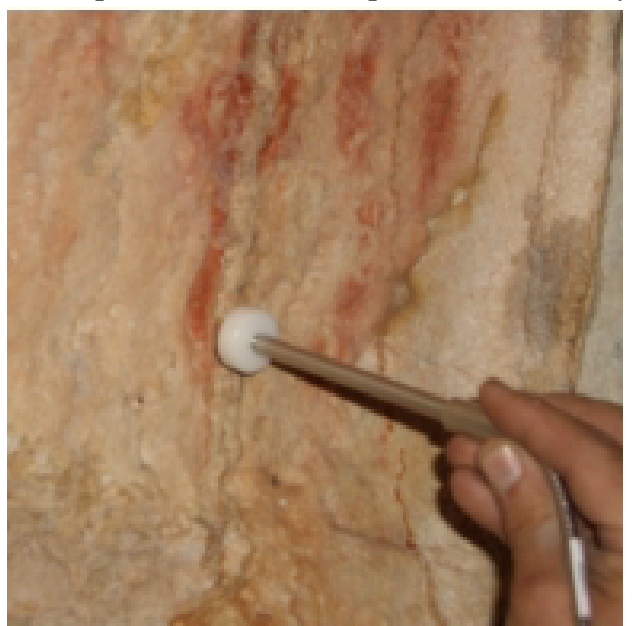

Fig. 1. Rock painting and the measurement procedure. of the cultural goods. On the other hand, an adequate conservation of them requires, in order to cause the minimal damage, to minimize the interaction of the artistic production exposed with the electromagnetic radiation ${ }^{[1-6]}$. In the case of the paintings, there are different factors which make that lighting plays a particularly important role. In any case, two major requirements should be satisfied: to minimize the damage and to obtain a good color reproduction.

The Murcielagos cave is placed in the Zuheros village in Córdoba (Spain). In this cave there are several important rock paintings. It is one of the most important Neolithic deposits of Andalucia, being the first one in which appears the art of the Upper Paleolithic. We have measured the spectral reflectance of paintings located in this cave. In this work we only consider one of them. The spectral reflectance of the rock in the surroundings of the paint has been also measured. In Figure 1 the painting and the measurement procedure are shown. From the measured spectral reflectances, a colorimetric characterization of the painting and the rock has been carried out (Section 2). In order to do it, we have considered that the rock paintings are illuminated with the spectral radiant distribution of a

Novel Optical Systems Design and Optimization XII, edited by R. John Koshel, G. Groot Gregory, Proc. of SPIE Vol. 7429, 742907 · @ 2009 SPIE · CCC code: 0277-786X/09/\$18 - doi: 10.1117/12.826503 
blackbody radiator at temperature $\mathrm{T}_{\mathrm{t}}=1850 \mathrm{~K}$. We have used this illuminant because this is the approximate temperature of a torch, which we assume that was the lighting source used by the original artists. In this way, the tristimulus values obtained with this illuminant are associated with the color stimulus perceived by the person who created the painting. In all this work we will consider these tristimulus values as the reference ones. Of course, when adopting this criterion, we have assumed that the color perception of the visual system of the human in the Upper Paleolithic was similar to that of the human at present. It should be pointed out that by the beginning of the Upper Paleolithic full behavioral modernity (is a term used in anthropology, archeology and sociology to refer to a list of traits that distinguish present day humans and their recent ancestors from both living primates and other extinct hominid lineages), including language, music and other cultural universals had developed.

The Section 3 of this work is devoted to analyze the colorimetric changes in the perception of the painting and rock when different sources of light are used: blackbody radiator at different temperatures, 38 illuminants recommended by the CIE [7] and a halogen lamp). The colorimetric changes are quantitatively evaluated by computing the distances in the CIELAB space between the color stimulus evoked to the observer when illuminating with the torch (reference tristimulus values) and that color stimulus generated when other different illuminant is used. This analysis will allow us to obtain a quantitative evaluation of the colorimetric behavior of the different sources of light when illuminating cave paintings. Proceeding according to conservation criteria, in order to determine the best source of light to be used in cave lighting is also necessary to analyze the damage that the different sources of light produce on the painting. By using the relative spectral response function (damage function) proposed by the CIE ${ }^{[6]}$, we have computed in Section 4 the effective irradiance damaging the painting for all the light sources previously used. The behavior of the different illuminants regarding to the produced damage is compared.

The paintings, object of this work, have a reddish tone fundamentally. This tonal simplicity will allow us to design a lighting source which spectral radiant power distribution minimizes the damage caused by the interaction of the pigment of the painting with the radiation without a significantly change in the color sensation as perceived by the original artists. In order to minimize the exposure of the pigment to the radiation, we will use low surrounding luminance levels. This fact also facilitate the visual adaptation of the spectator. In the last part of this work, starting with the approach introduced by Miller ${ }^{[8]}$, we propose an illuminant whose spectral distribution diminishes the damage by minimizing the absorption of radiation and optimizes the color perception of the paintings in this cave. The procedure followed in this study can be applied to optimize the lighting systems used when illuminating any other kind of art work.

\section{COLORIMETRIC CHARACTERIZATION OF THE PAINTING AND ROCK}

First of all, the spectral reflectance of the pigment has been measured in four different zones of the painting. The average curve will be denoted in the following as $\rho_{p}(\lambda)$. It has been also measured the spectral reflectance, $\rho_{r}(\lambda)$, of the wall in the surrounding of the painting in absence of pigments (rock). These measurements have been performed using an
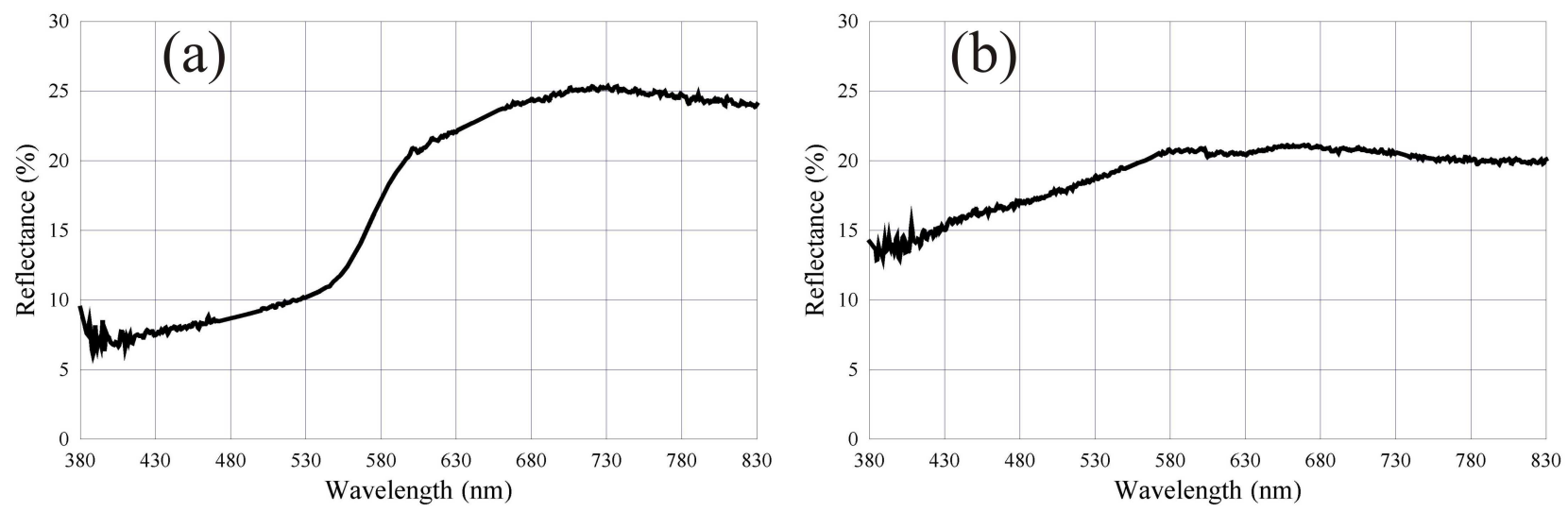

Fig. 2. Spectral reflectance. (a) painting and (b) rock. 
AvaSpec-2048-2 spectrophotometer in the spectral range from 400 to $750 \mathrm{~nm}$ with a step of $5 \mathrm{~nm}$. The curves representing the spectral reflectances are shown in Figure 2. In order to perform the necessary calculations, these spectral reflectances have been interpolated. As it has been pointed out in the previous Section, we assume that the lighting source used by the original artists was a torch, in such a way that it is considered that proper color reproduction is obtained when the painting is illuminated with the spectral radiant distribution of a blackbody radiator at temperature $\mathrm{T}_{\mathrm{t}}=1850 \mathrm{~K}$, which is given by ${ }^{[9]}$

$$
S_{T_{t}}(\lambda)=\frac{c_{1}}{\lambda^{5}} \frac{1}{e^{c_{2} /\left(\lambda T_{t}\right)}-1} \text { W.m. }
$$

The two ratiation constants are obtained from $c_{1}=2 \pi \mathrm{hc}^{2}=3.741832 \times 10^{-16} \mathrm{~W} \cdot \mathrm{m}^{2}$ and $\mathrm{c}_{2}=\mathrm{hc} / \mathrm{k}=1.438786 \times 10^{-2} \mathrm{~m} \cdot \mathrm{K}$. The tristimulus values of the painting when is lluminated with distribution (1) are computed as

$$
X_{i}^{T_{t}, p}=K_{T_{t}} \sum_{j=1}^{N} S_{T_{t}}\left(\lambda_{j}\right) \rho_{p}\left(\lambda_{j}\right) \hat{x}_{i}\left(\lambda_{j}\right) \Delta \lambda(\mathrm{i}=1,2,3),
$$

where $\hat{x}_{i}(\lambda)$ are the color-matching functions of the standard colorimetric observer CIE $1931,\left[\lambda_{1}, \lambda_{N}\right]=[400,750] \mathrm{nm}$ is the spectral interval in which the reflectances have been measured, and $\Delta \lambda=1 \mathrm{~nm}$. With this value, the number of wavelengths in which the visible spectrum is divided is $\mathrm{N}=350 \mathrm{~nm}$. In a similar way, the tristimulus values of the rock are

$$
X_{i}^{T_{t}, r}=K_{T_{t}} \sum_{j=1}^{N} S_{T_{t}}\left(\lambda_{j}\right) \rho_{r}\left(\lambda_{j}\right) \hat{x}_{i}\left(\lambda_{j}\right) \Delta \lambda(\mathrm{i}=1,2,3)
$$

In the last two equations, the constat $K_{T_{t}}$ is computed by considering as the white of reference the black body spectral distribution (1) in the following way ${ }^{[7]}$ :

$$
K_{T_{t}}=\frac{100}{\sum_{j=1}^{N} S_{T_{t}}\left(\lambda_{j}\right) \hat{x}_{2}\left(\lambda_{j}\right) \Delta \lambda}
$$

From the previous equations, we have computed the corresponding chromaticity coordinates, $\left(x_{1}^{p}, x_{2}^{p}\right)_{T_{t}}$ and $\left(x_{1}^{r}, x_{2}^{r}\right)_{T_{t}}$ of the painting and rock respectively when they are illuminated with the torch. The position of these chromaticities in the CIE 1931 chromaticity diagram are plotted in Figure 3.

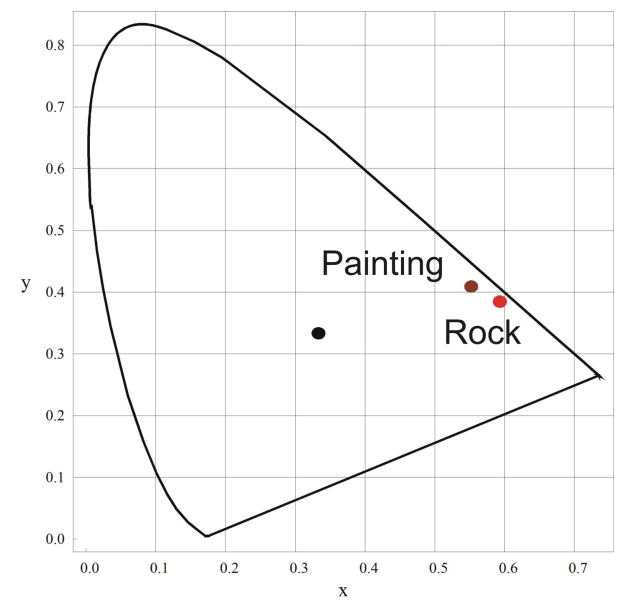

Fig. 3. Location in the CIE 1931 chromaticity diagram of the painting and rock when they are illuminated with the spectral distribution of a blackbody radiator at temperature $T_{T_{t}}=1580 \mathrm{~K}$. 
As it has been previously indicated, the tristimulus values (2) and (3) of the painting and rock respectively when illuminated with the spectral distribution (1), will be considered as the reference ones. All the variations of the colorimetric characteristics will be computed from these values.

\section{CHROMATIC DISPLACEMENTS}

In this Section we will analyze the variations of the color when the painting and rock are illuminated with a lighting source different to a torch. In the first sub-section we will consider the chromatic displacements when the source of light is a blackbody radiator and its temperature is variated. The second sub-section is devoted to the analysis of changes of color when the lighting sources are those standard illuminants recomended by the CIE.

\subsection{Variation of the colorimetric characteristics with the temperature of the blackboby radiator}

In the following, we will evaluate the variations of the color of painting and rock when they are illuminated with the spectral distribution of the blackbody radiation at temperatures differents from $T_{T_{t}}$. In this case, the spectral radiant power exitance is given by

$$
S(T, \lambda)=K(T) \frac{c_{1}}{\lambda^{5}} \frac{1}{e^{c_{2} /\left(\lambda T_{t}\right)}-1} \quad \mathrm{~W} \cdot \mathrm{m}^{-3},
$$

where $T$ is the temperature of the blackbody radiator. The value of $K(T)$ is chosen in such a way that the total radiant exitance is coincidental with that of the blackbody radiator at the temperature $T_{T_{t}}$ of the torch, i.e.,

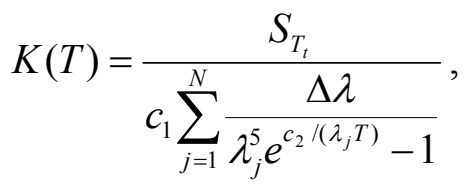

with

$$
S_{T_{t}}=\sum_{j=1}^{N} S_{T_{t}}\left(\lambda_{j}\right) \Delta \lambda
$$

In order to quantitatively compare the damage produced by different illuminants it is important that all of them have the same total radiant exitance, therefore the normalization from $\mathrm{K}(\mathrm{T})$ will play an important role when computing the damage that on the painting causes the lighting source. The tristimulus values of the painting and rock when illuminated with the spectral distribution (6) are

$$
X_{i}^{p}(T)=K_{T} \sum_{j=1}^{N} S\left(T, \lambda_{j}\right) \rho_{p}\left(\lambda_{j}\right) \hat{x}_{i}\left(\lambda_{j}\right) \Delta \lambda(\mathrm{i}=1,2,3)
$$

and

$$
X_{i}^{r}(T)=K_{T} \sum_{j=1}^{N} S\left(T, \lambda_{j}\right) \rho_{r}\left(\lambda_{j}\right) \hat{x}_{i}\left(\lambda_{j}\right) \Delta \lambda(\mathrm{i}=1,2,3)
$$

respectively, with

$$
K_{T}=\frac{100}{\sum_{j=1}^{N} S\left(T, \lambda_{j}\right) \hat{x}_{2}\left(\lambda_{j}\right) \Delta \lambda}
$$


With this choice of $\mathrm{K}_{\mathrm{T}}$, we have assumed that in each case the reference white stimulus is the source of light used to illuminate the painting. The tristimulus values have been calculated in the range of temperatures running from $\mathrm{T}=100 \mathrm{~K}$ to $\mathrm{T}=7000 \mathrm{~K}$ in steps $\Delta \mathrm{T}=100 \mathrm{~K}$. From (8) and (9) we have compute de chromaticity coordinates $\left(x_{1}^{p}(T), x_{2}^{p}(T)\right)$ and $\left(x_{1}^{r}(T), x_{2}^{r}(T)\right)$ associated with the painting and the rock respectively when the source of light used is the distribution (5). The variation of the chromaticity coordinates with the temperature of the blackbody radiator used is shown in Figure 4.

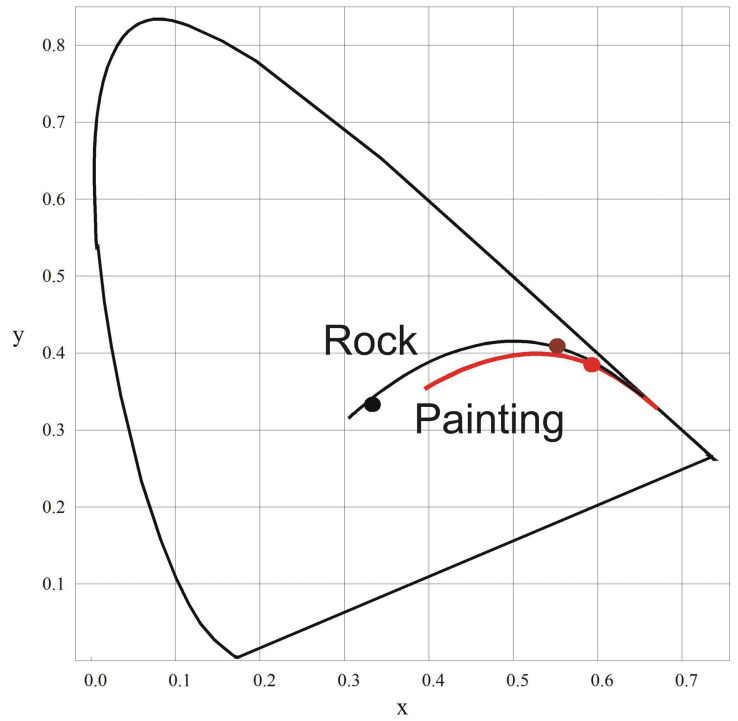

Fig. 4. Chromaticity diagram CIE 1931 showing the parametric curves $\left(x_{1}^{p}(T), x_{2}^{p}(T)\right) \quad$ and $\left(x_{1}^{r}(T), x_{2}^{r}(T)\right) \quad$ which provide the variation of the chromaticity coordinates of the painting and rock respectively as a function of the temperature of the blackbody radiator used as lighting source.

The variation of the chromaticity coordinates exhibit an expected behavior. When the temperature of the lighting source is increased, the chromaticity coordinates of both, painting and rock, approache the center of the chromaticity diagram. It should be pointed out that the line describing the variation of the chromaticity coordinates of the rock is very similar to the Planckian locus. However, the painting has always a more reddish tone that the Planckian locus. This behavior is an expected one if we analyze the spectral reflectances represented in Figure 2.

In order to quantitatively compute the differences between the color stimulus obtained when the painting and rock are illumianted with a torch a that color stimulus perceived when the lighting source is a balckbody radiator at temperature T, all the color stimuli specified in the CIE 1931 system have been transformed to the CIELAB color space ${ }^{[7]}$. As it is well known, in this space the coordinates $\left(\mathrm{L}^{*}, \mathrm{a}^{*} \cdot \mathrm{b}^{*}\right)$ of a color stimulus are related with the corresponding tristimulus values $\left(\mathrm{X}_{1}, \mathrm{X}_{2}, \mathrm{X}_{3}\right)$ in the CIE 1931 system by the following relations:

$$
\begin{gathered}
L^{*}=116\left(\frac{X_{2}}{X_{2, w}}\right)^{1 / 3}-16, \\
a^{*}=500\left[\left(\frac{X_{1}}{X_{1, w}}\right)^{1 / 3}-\left(\frac{X_{2}}{X_{2, w}}\right)^{1 / 3}\right], \\
b^{*}=200\left[\left(\frac{X_{2}}{X_{2, w}}\right)^{1 / 3}-\left(\frac{X_{3}}{X_{3, w}}\right)^{1 / 3}\right],
\end{gathered}
$$

and

where $\left(\mathrm{X}_{1, \mathrm{w}}, \mathrm{X}_{2, \mathrm{w}}, \mathrm{X}_{3, \mathrm{w}}\right)$ are the tristimulus values of of the light source used in each case with $\mathrm{X}_{2, \mathrm{w}}=100$. By taking into account this transformation, from the tristimulus values (2) and (3), we have computed the coordinates $\left(L_{T_{t}}^{p}, a_{T_{t}}^{p}, b_{T_{t}}^{p}\right)$ and $\left(L_{T_{t}}^{r}, a_{T_{t}}^{r}, b_{T_{t}}^{r}\right)$ in the CIELAB space for the painting and the rock respectively when they are 
illuminated with the torch. In a similar way, using the tristimulus values (8) and (9), the CIELAB coordinates $\left(L^{p}(T), a^{p}(T), b^{p}(T)\right)$ and $\left(L^{r}(T), a^{r}(T), b^{r}(T)\right)$ for the painting and rock when a blackbody radiator at temperature $\mathrm{T}$ is used as lighting source are also computed.

In the CIELAB space, the Euclidean distances can be used to represent approximately the perceived magnitude of color differences between two color stimuli. Thus, the colorimetric differences between the painting when illuminated by the torch and when the lighting source is the blackbody radiator at temperature $T_{t}$, can be computed as follows:

$$
\Delta E_{T_{t}}^{p}(T)=\left[\left(L_{T_{t}}^{p}-L^{p}(T)\right)^{2}+\left(a_{T_{t}}^{p}-a^{p}(T)\right)^{2}+\left(b_{T_{t}}^{p}-b^{p}(T)\right)^{2}\right]^{1 / 2} .
$$

In a similar way, the color difference between the rock illuminated by the torch and illuminated by the blackbody radiator a temperature $\mathrm{T}$ is given by

$$
\Delta E_{T_{t}}^{p}(T)=\left[\left(L_{T_{t}}^{r}-L^{r}(T)\right)^{2}+\left(a_{T_{t}}^{r}-a^{r}(T)\right)^{2}+\left(b_{T_{t}}^{r}-b^{r}(T)\right)^{2}\right]^{1 / 2} .
$$

The curves which describe the variation of the color differences as a function of the temperature are represented in Figure 5.
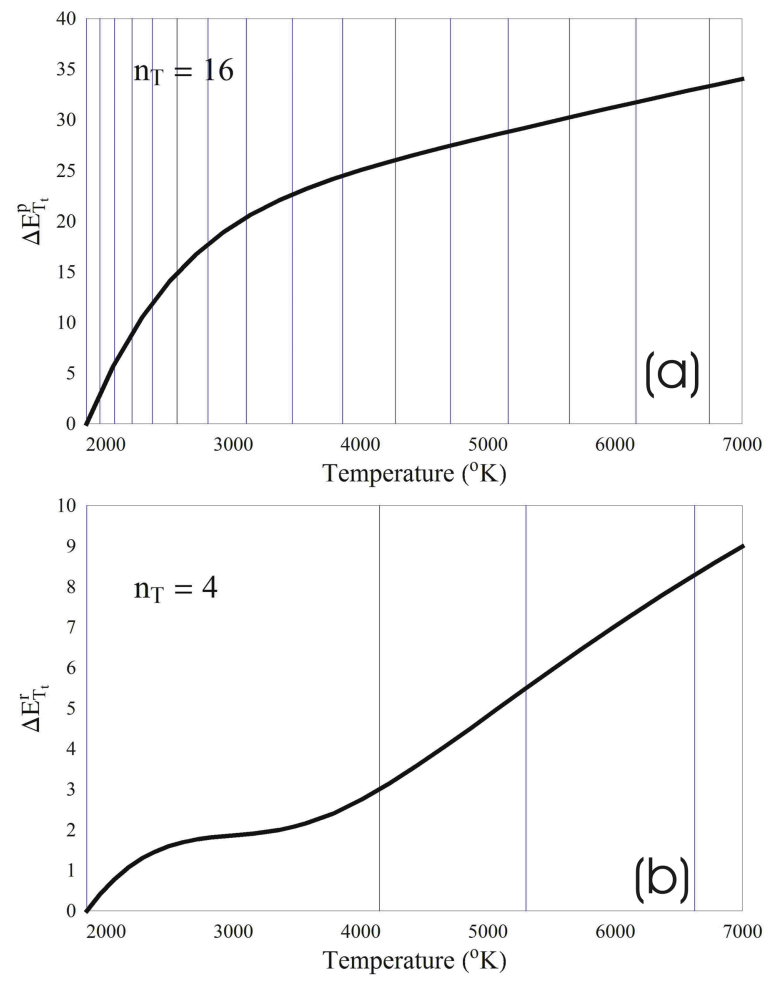

Fig. 5. Distance between the color stimulus evoked when illuminating with the torch and the color stimulus generated when the lighting source is a blackbody radiator at temperature T. (a) Painting and (b) Rock. The vertical stright lines show intervals of temperature in which the distance is three CIELAB units. In each case, the range of temperatures between two straigh lines provides color stimuli undistinguishable among them. Then number total of intervals containing temperatures providing undistinguishable stimili is (a) $n_{t}=16$ for the painting and (b) $n_{t}=4$ for the rock.

As it is shown in this Figure, for a given temperature, the distance between the color estimulus generated when illuminating with the corresponding blackbody radiator and the color estimulus associated with the torch is always higher in the case of the painting. It means that the difference of color perceived when the lighting source is different that a torch is always lesser when illuminating the rock. In this way, it becomes obvious that to illuminate the rock is a less 
critical task that to illuminate the pigment. The color differences due to the use of an illuminant different to a torch are perceived with more intensity in the case of the painting.

Usually it is assumed that a color differences E lesser than three CIELAB units are associated with color stimuli which are undistinguishable between them. Thus, in Figure 5, the interval between two consecutive straight lines is associated with temperatures that evokes the same color sensation to a standard observer. In this way, the first interval in both graphics corresponds to temperatures of the blackbody radiator used as illuminant which provides color stimuli undistinguishable from that generated when illuminating with a torch. From a colorimetric point of view, it is equivalent to illuminate the painting or rock with a torch that to do it using a blackbody radiator whose temperature belongs to the firs interval in each graphic. This first interval is considerably lesser in the case of the painting. This fact means that the rage of variation of temperatures of the blackbody radiator in which the color perception is similar to that evoked by a torch is reduced. Nevertheless, in the case of the rock it is possible to use a blackbody radiator with temperature belonging to the interval between $1000 \mathrm{~K}$ and $4000 \mathrm{~K}$ without change in the color perception with regard to the color stimulus evoked when using as lighting source the torch. In the case of the painting, there are $n_{t}=16$ intervals containing temperatures which provides undistinguishable color stimuli. However, in the case of the rock this number of intervals is $\mathrm{n}_{\mathrm{t}}=4$. As a consequence of this fact we can use a larger interval of different temperatures without change in the color perception in the case of the rock that in the case of the painting.

\subsection{Variation of the colorimetric characteristics when using different standar illuminants.}

Now, we will analyze the change of the color of the painting and rock when they are illuminated with a set of 40 different illuminants: : the 38 illuminants recommended by the CIE plus a xenon and halogen lamps. The numbering used and the description of the illuminants are provided in Table I.

Table 1. Numbering and description of the illuminants. The number in columns one and three is that assigned by us to each source of light. The nomenclature is that proposed by the CIE. 7-12: Standard fluorescent lamps; 13-15: Broad-band fluorescent lamps; 16-18: Narrow-band fluorescent lamps; 1921: Standard halophosphate lamps; 22-24: DeLuxe type lamps; 25-29: Three-band fluorescent lamps; 30-32: Multi-band fluorescent lamps; 33: D65 simulator lamp; 34: High pressure discharge lamp; 35 : Standard high pressure sodium lamp; 36-38: High pressure metal halide lamp.

\begin{tabular}{|c|l|c|l|}
\hline$\alpha$ & \multicolumn{1}{|c|}{ Source } & $\alpha$ & \multicolumn{1}{|c|}{ Source } \\
\hline 1 & A illuminant & 21 & FL3.3 \\
\hline 2 & D65 Illuminant & 22 & FL3.4 \\
\hline 3 & C illuminant & 23 & FL3.5 \\
\hline 4 & D50 Illuminant & 24 & FL3.6 \\
\hline 5 & D55 Illuminant & 25 & FL3.7 \\
\hline 6 & D75 Illuminant & 26 & FL3.8 \\
\hline 7 & FL1 & 27 & FL3.9 \\
\hline 8 & FL2 & 28 & FL3.10 \\
\hline 9 & FL3 & 29 & FL3.11 \\
\hline 10 & FL4 & 30 & FL3.12 \\
\hline 11 & FL5 & 31 & FL3.13 \\
\hline 12 & FL6 & 32 & FL3.14 \\
\hline 13 & FL7 & 33 & FL3.15 \\
\hline 14 & FL8 & 34 & HP1 \\
\hline 15 & FL9 & 35 & HP2 \\
\hline 16 & FL10 & 36 & HP3 \\
\hline 17 & FL11 & 37 & HP4 \\
\hline 18 & FL12 & 38 & HP5 \\
\hline 19 & FL3.1 & 39 & Xenon lamp \\
\hline 20 & FL3.2 & 40 & Halogen lamp \\
\hline
\end{tabular}

Let $S_{\alpha}(\lambda)$ be the spectral radiant power distribution of the illuminant $\alpha(\alpha=1,2, \ldots, 40)$. This distribution has been normalized in such a way that the total radiant exitance is coincidental with that of the blackbody radiator at the 
temperature $T_{T_{t}}$ of the torch. The tristimulus values obtained for the painting and rock when the illuminant is used as lighting source are respectively

$$
X_{i}^{p, \alpha}(T)=K_{\alpha} \sum_{j=1}^{N} S_{\alpha}\left(\lambda_{j}\right) \rho_{p}\left(\lambda_{j}\right) \hat{x}_{i}\left(\lambda_{j}\right) \Delta \lambda(\mathrm{i}=1,2,3)
$$

and

$$
X_{i}^{r, \alpha}(T)=K_{\alpha} \sum_{j=1}^{N} S_{\alpha}\left(\lambda_{j}\right) \rho_{r}\left(\lambda_{j}\right) \hat{x}_{i}\left(\lambda_{j}\right) \Delta \lambda(\mathrm{i}=1,2,3)
$$

with

$$
K_{\alpha}=\frac{100}{\sum_{j=1}^{N} S_{\alpha}\left(\lambda_{j}\right) \hat{x}_{2}\left(\lambda_{j}\right) \Delta \lambda}
$$

The chromaticity coordinates obtained for all the illuminants are represented in Figure 6 in the CIE 1931 chromaticity diagram.
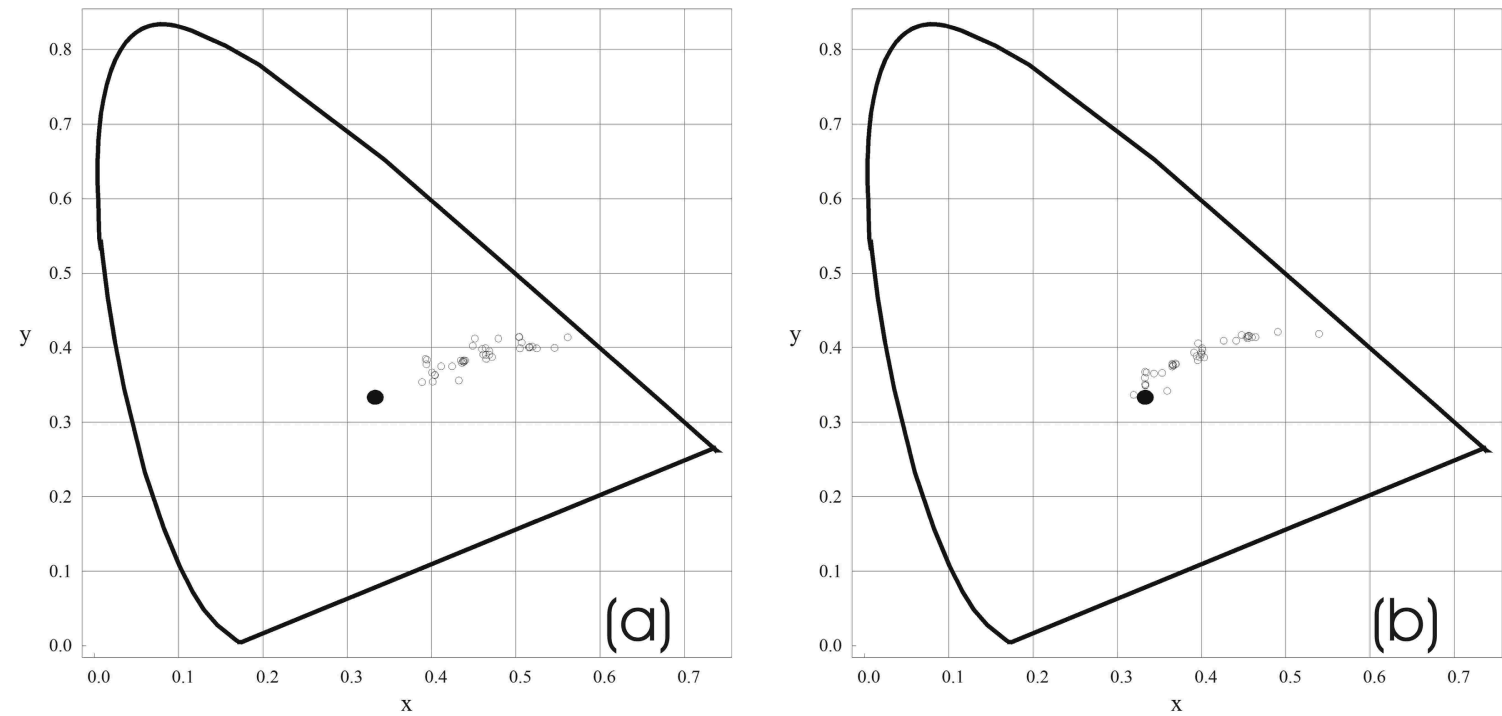

Fig. 6. CIE 1931 chromaticity diagram showing the chromaticity coordinates obtained when the 40 illuminants listed in Table 1 are used as lighting sources. (a) Chromaticity coordinates for the painting. (b) Chromaticity coordinates for the rock.

It becomes obvious that the chromaticities of the painting, or rock, are very different between them when different illuminants are used. This result points out how the color perception of the work art strongly depends on the illuminant used as lighting source. When designing a lighting sistem for cave paintings, we must be careful in the choice of the source of light. In our case we must select the illuminant which minimizes the differences with the color perceived when the painting is illuminated with a torch. In order to choice a such illuminant, we must to quantitatively stimate the color differences between the color reproduced with a given illuminant and the color that we like to reproduce. It will be done in the following.

From the previous results, and taking into account Equations (11) we have transformed the tristimulus values in the CIE 1931 system to the CIELAB system. We will denote as $\left(L_{\alpha}^{p}, a_{\alpha}^{p}, b_{\alpha}^{p}\right)$ and $\left(L_{\alpha}^{r}, a_{\alpha}^{r}, b_{\alpha}^{r}\right)$ the coordinates in this spaces of 
the painting and rock respectively when the lighting source is the illuminant $\alpha$. In order to quantitatively evaluate the colorimetric behavior of the forty illuminants considered in this work, we hae computed the distance in the CIELAB space between the color stimulus associated with the illuminant $\alpha$ and the color stimulus obtained when the lighting source is the torch. This distances are given by

$$
\Delta E_{\alpha}^{p}=\left[\left(L_{\alpha}^{p}-L_{T_{t}}^{p}\right)^{2}+\left(a_{\alpha}^{p}-a_{T_{t}}^{p}\right)^{2}+\left(b_{\alpha}^{p}-b_{T_{t}}^{p}\right)^{2}\right]^{1 / 2}
$$

and

$$
\Delta E_{\alpha}^{r}=\left[\left(L_{\alpha}^{r}-L_{T_{t}}^{r}\right)^{2}+\left(a_{\alpha}^{r}-a_{T_{t}}^{r}\right)^{2}+\left(b_{\alpha}^{r}-b_{T_{t}}^{r}\right)^{2}\right]^{1 / 2},
$$

for the painting and rock respectively. The values obtained for these distances are represented in Figure 7. A quickly inspection of this Figure points out how the distances are considerably larger for the painting than for the rock. This

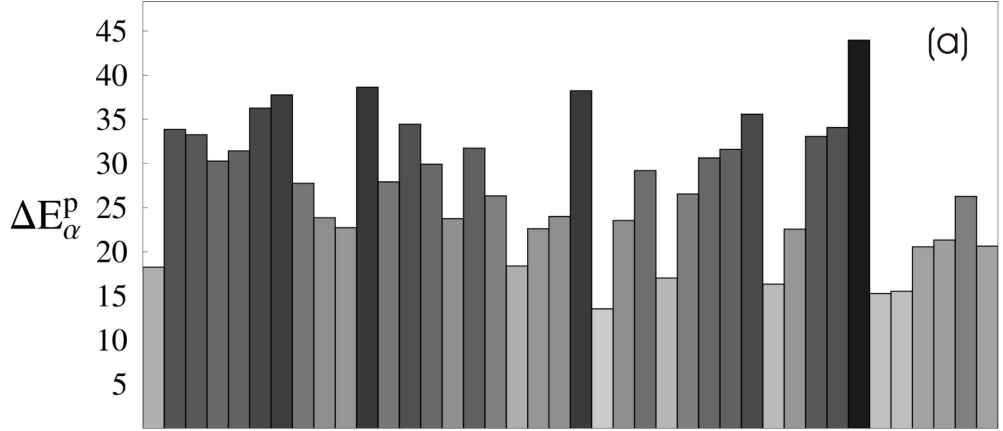

$2 \quad 4 \quad 6 \quad 8 \quad 10121416182022242628303234363840$ Number of the source $\alpha$

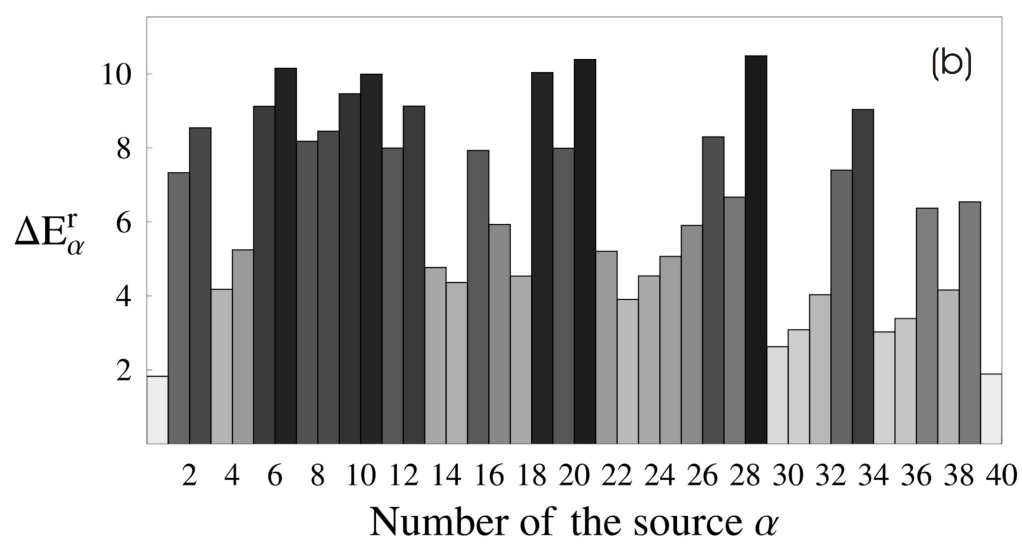

Fig. 7. Values of the distance between the color stimulus obtained when illuminating with the illuminant $\alpha$ and the stimulus generated when the lighting source is the torch. (a) Distances for the painting, given by Equation (17). (b) Distances for the rock, given by Equation (18). result confirms again the previous one: the lighting of the rock is less restrictive that the lighting of the painting. When using any of the considered illuminants, the color differences perceived for the rock are always lesser than for the painting. It should be pointed out that, when the painting is illuminated with any of the forty considered sources of light, all the values of the distances are grater than three CIELAB units. In this way, the colour perceived by an standard observer is always different, with the illuminants listed in Table 1, than that perceived by the original artists. When the painting is considered, the larger value of the distance is obtained for the illuminant 34 (High pressure discharge lamp HP1) with $\Delta E_{\alpha}^{p}=44$ CIELAB units and the lesser value is reached for the illuminant 22 (DeLuxe type lamp FL3.4) with $\Delta E_{\alpha}^{p}=14$ CIELAB units. In any case, this distance is very large, in such a way that the corresponding illuminant does not provide an adequate color perception. The spectral radiant power distribution of the illuminants 34 (maximum of distance) and 22 (minimum of distance) are plotted in Figure 8. However, for the rock can be used some of the sources of light without change, or with a minimal change, in the color perception (distances lesser o near to three). These sources of light are the

following: 1 (A illuminant), 23 (DeLuxe type lamp F13.5), 30 (Multi-band fluorescent lamp F13.12), 31 (Multi-band fluorescent lamp F13.13), 35 (Standard high pressure sodium lamp HP2), and 36 (High pressure metal halide lamp HP3). From the previous analysis it can be concluded that, in the case of our painting, it is not possible to obtain an adequate color reproduction by using the considered illuminants. In any case, Table 1 and Figure 7 can help us in order to choice, or avoid, a source of light in caves lighting. The procedure followed in this Section can be applied to optimise, from a colorimetric point of view, the design of any other project of cultural goods lighting. Of course the results will depend on 

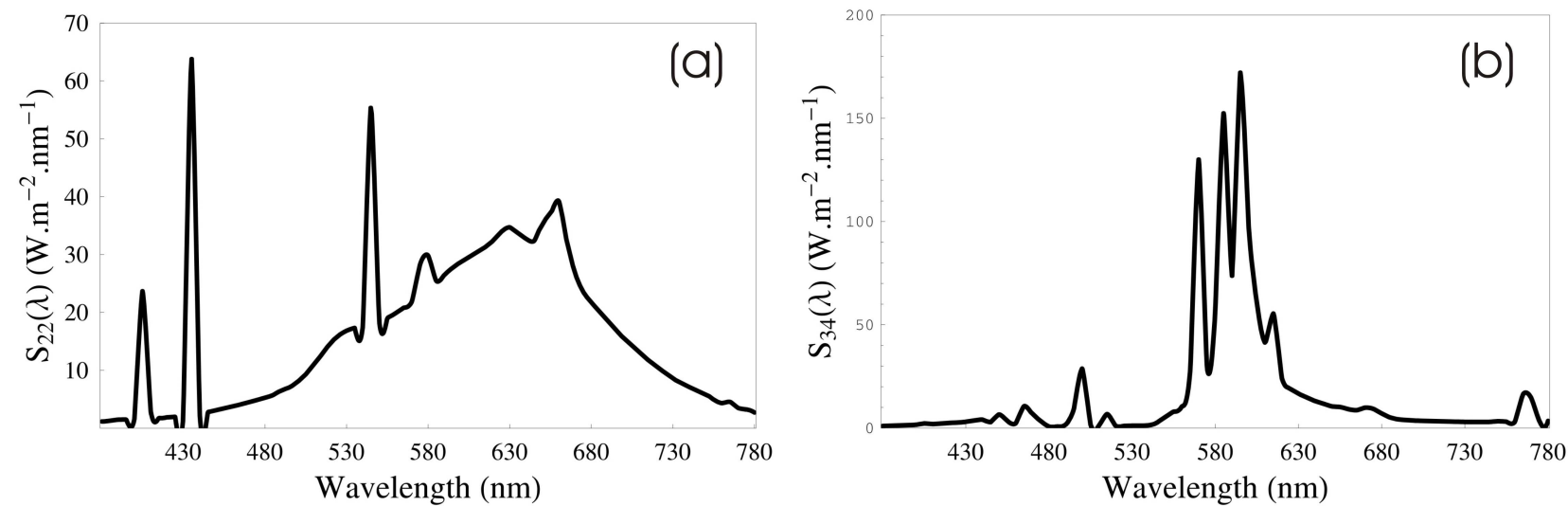

Fig. 8. Spectral radiant power distribution of (a) the illuminant 22 (DeLuxe type lamp FL3.4) which minimizes the distance from the color stimulus produced when the torch illuminates the painting and (b) the illuminant 34 (High pressure discharge lamp HP1) which provides the maximum of the distance.

the considered illuminants and on the spectral reflectance of the objects to be illuminated. It becomes also necessary to adequately choice the reference illuminant from which the distances of the perceived colors should be minimized.

\section{ANALYSIS OF THE DAMAGE}

As it has been pointed out, in order to design an adequate lighting system for cave painting, or any other cultural good, it becomes necessary to analyze at the same time the color reproduction and the damage that the radiation produces on the illuminated object. The first of these task has been carried out in the previous Section. In the following, we will compute the irradiance which produces damage for each one of the previously considered illuminants. We will refer to this irradiance as "damage effective irradiance". According with the recommendations provided by the CIE ${ }^{[6]}$, this damage effective irradiance is given by

$$
E_{d m}=\sum_{j=1}^{N} S\left(\lambda_{j}\right) D\left(\lambda_{j}\right) \Delta \lambda,
$$

where $S(\lambda)$ is the spectral irradiance of the which is being tested and

$$
D(\lambda)=e^{-a\left(\lambda-\lambda_{0}\right)}
$$

is the damage factor proposed by the CIE. In the last expression the value of $\lambda_{0}$ is $300 \mathrm{~nm}$ and a is a constant whose value is $0.0115 \mathrm{~nm}^{-1}$ when oiled paints are considered (this is the more similar case to that of the cave paintings). By introducing in expression (19) the spectral distribution (5) we have computed the damage effective irradiance as a function of the temperature of the blackbody radiator used as lighting source, $E_{d m}(T)$. Function $E_{d m}(T)$ is a monotonous increasing function of the temperature and, for the range of temperatures considered in this work (1000 $\mathrm{K}$ to $7000 \mathrm{~K}$ ), the minimum and maximum values are $47 \mathrm{~W} \cdot \mathrm{m}^{-2}$ and $985 \mathrm{~W} \cdot \mathrm{m}^{-2}$ respectively. It should be pointed out that the damage effective irradiance produced by the torch is $E_{d m}(1850)=112 \mathrm{~W} . \mathrm{m}^{-2}$.

In a similar way, when the $S_{\alpha}(\lambda)$ spectral distribution associated with the illuminant $\alpha$ is used in expression (19) we obtain the damage effective irradiance, $E_{d m}^{\alpha}$, for the corresponding illuminant. The results obtained are represented in Figure 9. The minimum value $\left(E_{d m}^{1}=338 \mathrm{~W} \cdot \mathrm{m}^{-2}\right)$ is reached for the A illuminant $(\alpha=1)$ and the maximum $\left(E_{d m}^{21}=1065 \mathrm{~W} \cdot \mathrm{m}^{-2}\right)$ is obtained for the Standard halophospate lamp FL3.3 $(\alpha=21)$. In any case all the illuminants produce a damage effective irradiance higher than that of the torch. 


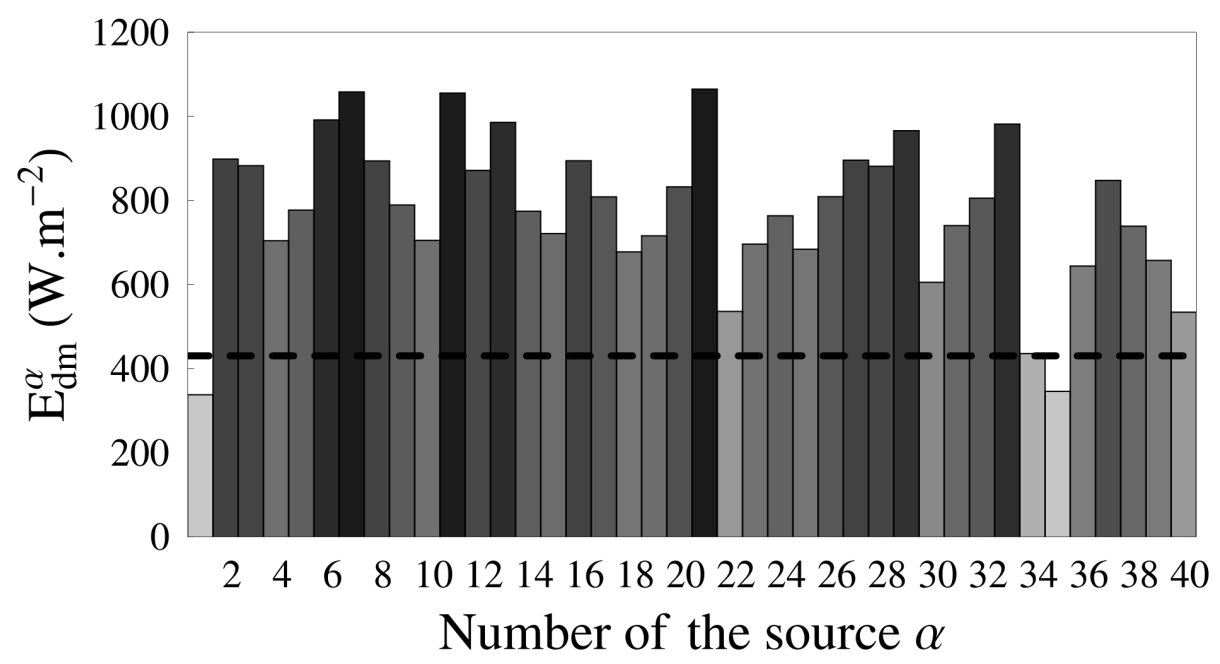

Fig. 9. Damage effective irradiance for the forty illuminants considered in this work. The dashed line corresponds to the value, $E_{d m}^{o p t}$, obtained for the optimal illuminant.

The illuminants for which the damage is lesser are those with $\alpha=1, \alpha=35$, and $\alpha=34$, but, as it is shown in Figure 7 (a), they have too large values of the distance (17) in order to provide and adequate color perception for the painting. For these illuminants the distances $\Delta E_{\alpha}^{p}$ are 18,15 , and 44 CIELAB units respectively. With these values it is not possible to obtain an adequate chromatic reproduction.

When computing the damage effective irradiance according expressions (19) and (20), the results obtained do not depend on the reflectance of the illuminated object. It seems an evident fact that the damage on the object should be depend on its reflectance. In order to take into account this fact, we have consider the criterion proposed by Miller ${ }^{[8]}$. According to it, the absorption of electromagnetic radiation is minimized when the spectral radiant power distribution is coincidental with the spectral reflectance of the object to be illuminated. We will refer to a such source of light as the "Miller optimal illuminant". In our case this the spectral distribution of this lighting source must be equal to the spectral reflectance of the painting, i.e.,

$$
S_{\text {opt }}(\lambda)=\rho_{p}(\lambda)
$$

When we introduce this distribution in expression (19), we obtain that the corresponding damage effective irradiance is $E_{d m}^{o p t}=450 \mathrm{~W} \cdot \mathrm{m}^{-2}$. This value is indicated in Figure 9 by the dashed line. From equation (17), we have also computed the distance $\Delta E_{o p t}^{p}$ which provides a measure of the perceived color difference between the stimulus generated when the painting is illuminated with the torch and that produced when the source of lighting is the Miller optimal illuminant. The value obtained is $\Delta E_{o p t}^{p}=10$ CIELAB units. Although we can see in Figure 9 that the value of the damage produced by the Miller optimal illuminant is slightly higher than that generated by the three previously mentioned illuminants $(\alpha=1$, $\alpha=35$, and $\alpha=34$ ), we can appreciate in Figure 7(b) that the distance $\Delta E_{o p t}^{p}$ is substantially lesser than all the distances $\Delta E_{\alpha}^{p}$ computed for the forty illuminants considered in this work. From this, we can conclude that, although there are values of the damage effective irradiance lesser than that obtained with the Miller optimal illuminant, the last one is the lighting source providing a better color reproduction. 


\section{CONCLUSSIONS}

In this work we have analysed the change of the color sensation produced when different sources of light are used to illuminate a cave painting. We have also study the damage produced on the painting as a function of the illuminant used. As a consequence of this analysis, we have proposed as source of lighting an optimal illuminant. This illuminant provides a very low values of the damage effective irradiance and a better color perception than that obtained with all the other considered illuminants.

The procedure followed in this work can be systematized in order to be applied to optimise the color perception and the produced damage in the design of any other lighting project applied to cultural goods. Of course, the results obtained in each project will be different and they will depend on the considered illuminants and on the spectral reflectance of the objects to be illuminated.

\section{REFERENCES}

[1] Cuttle, C., "Damage to museum objects due to light exposure", Light. Res. Tech., 28(1), 1 -9 (1996).

[2] Cuttle C., "Lighting works of art for exhibition and conservation", Light.Res.Tech. 20(2), 43-53 (1988).

[3] Hoon, K. and Hong-Bum, K., "New evaluation method for the lightfastness fo colored papers by radiant energy", J. Illum. Eng. Soc., 17-24, winter 2000.

[4] Garcia, I., [La conservación preventiva y la exposición de objetos y obras de arte], KR (1999).

[5] Schaeffer, T., "Effects of Light on materials in collections", Getty conservation Institute, 2001.

[6] CIE. Control of damage to museum objects by optical radiation. CIE Publication No 157. Vienna: CIE Central Bureau; 2004.

[7] CIE. Colorimetry. CIE Publication No 15. Vienna: CIE Central Bureau; 2004.

[8] Miller, J.V., "Evaluating fading characteristic of light sources", Nouvir Research Co., Pasadena (1993).

[9] Wyszecky G. and Stiles W.S., [Color Science. Concepts and methods, Quantitative Data and Formulae], second edition, Jhon Wiley \& sons, New York (2000). 